\title{
Density Functional Theory Calculations of Lithium Adsorption and Insertion to Defect-Free and Defective Graphene
}

\author{
Yasuharu Okamoto
}

The IoT Devices Research Laboratories, NEC Corporation, 34 Miyukigaoka, Tsukuba,

\author{
Ibaraki, 305-8501 Japan
}

\section{Supporting Information}

Figure S1: Snapshot of molecular dynamics simulation

Movie S2: Movie file of molecular dynamics simulation 

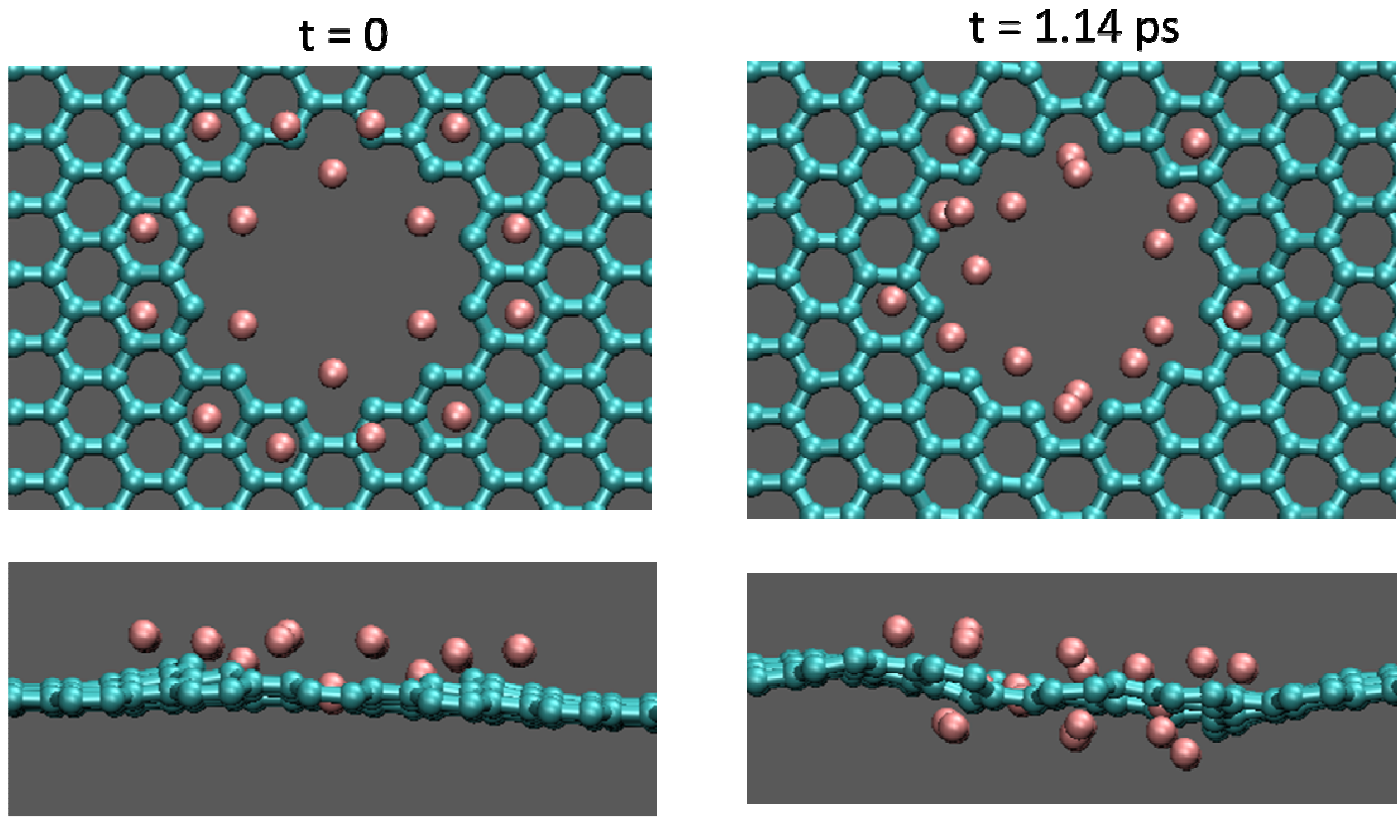

Figure S1: Snapshot of molecular dynamics simulation of $\mathrm{Li}$ penetration of $\mathrm{V}_{\mathrm{C} 24}$ vacancy at $500 \mathrm{~K}$ (controlled by velocity scaling method). Six Li atoms are inserted into $\mathrm{V}_{\mathrm{C} 24}$ and another $12 \mathrm{Li}$ atoms are located on the one side of the graphene sheet at $\mathrm{t}=0$ (left). Some Li atoms penetrate through the vacancy at $\mathrm{t}=1.14$ ps (right). Pink and sky blue balls stand for $\mathrm{Li}$ and $\mathrm{C}$, respectively. 